\title{
Assessment of three risk evaluation systems for patients aged $\geq 70$ in East China: performance of SinoSCORE, EuroSCORE II and STS risk evaluation system
}

\author{
Lingtong Shan ${ }^{1}$, Wen Ge ${ }^{2}$, Yiwei Pu ${ }^{1}$, Hong Cheng ${ }^{3}$, Zhengqiang Cang ${ }^{1}$, Xing Zhang ${ }^{1}$, Qifan Li $^{1}$, \\ Anyang Xu ${ }^{4}$, Qi Wang ${ }^{1}$, Chang Gu ${ }^{5}$, Yangyang Zhang ${ }^{\text {Corresp. } .6,7,8}$ \\ ${ }^{1}$ The First Clinical Medical College, Nanjing Medical University, Nanjing, China \\ 2 Department of Cardiothoracic Surgery, Shuguang Hospital affiliated to Shanghai University of TCM, Shanghai, China \\ 3 Department of Neurology, Jiangsu Province People's Hospital, the First Affiliated Hospital of Nanjing Medical University, Nanjing, China \\ 4 Department of Chronic and Noncommunicable Disease, Shanghai Changning District Center for Disease Control and Prevention, Shanghai, China \\ 5 Department of Thoracic Surgery, Shanghai Chest Hospital, Shanghai JiaoTong University, Shanghai, China \\ 6 Key Laboratory of Arrhythmias of the Ministry of Education of China, East Hospital, Tongji University School of Medicine, Shanghai, China \\ 7 Department of Cardiovascular Surgery, East Hospital, Tongji University School of Medicine, Shanghai, China \\ 8 Department of Thoracic and Cardiovascular Surgery, Jiangsu Province People's Hospital, The First Affiliated Hospital of Nanjing Medical University, \\ Nanjing, China \\ Corresponding Author: Yangyang Zhang \\ Email address: zhangyangyang_wy@vip.sina.com
}

Objectives: To assess and compare the predictive ability of three risk evaluation systems (SinoSCORE, EuroSCORE II and STS risk evaluation system) in patients aged $\geq 70$, and who underwent coronary artery bypass grafting (CABG) in East China. Methods: Three risk evaluation systems were applied to 1946 consecutive patients who underwent isolated CABG from January 2004 to September 2016 in two hospitals. Patients were divided into two subsets according to their age: elderly group (age $\geq 70$ ) with a younger group (age $<70$ ) used for comparison. The outcome of interest in this study was in-hospital mortality. The entire cohort and subsets of patients were analyzed. The calibration and discrimination in total and in subsets were assessed by the Hosmer-Lemeshow and the $\mathrm{C}$ statistics respectively. Results: Institutional overall mortality was $2.52 \%$. The expected mortality rates of SinoSCORE, EuroSCORE II and STS risk evaluation system were $0.78(0.64) \%$, $1.43(1.14) \%$ and $0.78(0.77) \%$, respectively. SinoSCORE achieved the best discrimination (the area under the receiver operating characteristic curve $(A \cup C)=0.829$ ), followed by STS risk evaluation system ( $A U C=0.790)$ and EuroSCORE II $(A U C=0.769)$ in the entire cohort. In the elderly group, the observed mortality rate was $4.82 \%$ while it was $1.38 \%$ in the younger group. SinoSCORE ( $A \cup C=0.829$ ) also achieved the best discrimination in the elderly group, followed by STS risk evaluation system $(A U C=0.730)$ and EuroSCORE II $(A \cup C=0.640)$ while all three risk evaluation systems all had good performances in the younger group. SinoSCORE, EuroSCORE II and STS risk evaluation system all achieved positive calibrations in the entire cohort and subsets. Conclusion: The performance of the 
three risk evaluation systems was not ideal in the entire cohort. In the elderly group, SinoSCORE appeared to achieve better predictive efficiency than EuroSCORE II and STS risk evaluation system. 
1

2

3

4

\section{evaluation system}

Assessment of three risk evaluation systems for patients aged $\geq 70$ in East China: performance of SinoSCORE, EuroSCORE II and STS risk

\section{(1)}

Lingtong $\mathrm{Shan}^{1}{ }^{\Delta}$, Wen $\mathrm{Ge}^{2 \Delta}$, Yiwei $\mathrm{Pu}^{1}{ }^{1}$, Hong Cheng $^{3}{ }^{3}$, Zhengqiang Cang ${ }^{1}$, Xing Zhang ${ }^{1}$, Qifan $\mathrm{Li}^{1}$, Anyang $\mathrm{Xu}^{4}$, Qi Wang ${ }^{1}$, Chang $\mathrm{Gu}^{5}$, Yangyang Zhang ${ }^{6,7,8 *}$
${ }^{1}$ The First Clinical Medical College of Nanjing Medical University, Nanjing, China
${ }^{2}$ Department of Cardiothoracic Surgery, Shuguang Hospital affiliated to Shanghai University of TCM,
Shanghai, China

${ }^{3}$ Department of Neurology, Jiangsu Province People's Hospital, the First Affiliated Hospital of Nanjing

Medical University, Nanjing, China

${ }^{4}$ Shanghai Changning District Center for Disease Control and Prevention, Shanghai, China

${ }^{5}$ Department of Thoracic Surgery, Shanghai Chest Hospital, Shanghai JiaoTong University, Shanghai,

(1)

China

${ }^{6}$ Department of Cardiovascular Surgery, East Hospital, Tongji University School of Medicine, Shanghai,

15

\section{China}

${ }^{7}$ Key Laboratory of Arrhythmias of the Ministry of Education of China, East Hospital, Tongji University School of Medicine, Shanghai, China

${ }^{8}$ Department of Thoracic and Cardiovascular Surgery, Jiangsu Province People's Hospital, The First Affiliated Hospital of Nanjing Medical University, Nanjing, China 
$21 *$ Address for correspondence:

22 Yangyang Zhang, PhD, MD, Department of Cardiovascular Surgery, Shanghai East Hospital, East

23 Hospital Affiliated to Tongji University, 150 Jimo Road, Shanghai 200120, China. Tel: +86 13818132320,

24 E-mail: zhangyangyang_wy@vip.sina.com 


\section{ABSTRACT}

Objectives: To assess and compare the predictive ability of three risk evaluation systems (SinoSCORE,

EuroSCORE II and STS risk evaluation system) in patients aged $\geq 70$, and who underwent coronary artery bypass grafting $(\mathrm{CABG})$ in East China.

Methods: Three risk evaluation systems were applied to 1946 consecutive patients who underwent isolated CABG from January 2004 to September 2016 in two hospitals. Patients were divided into two subsets according to their age: elderly group (age $\geq 70$ ) with a younger group (age $<70)$ used for comparison. The outcome of interest in this study was in-hospital mortality. The entire cohort and subsets of patients were analyzed. The calibration and discrimination in total and in subsets were assessed by the Hosmer-Lemeshow and the $\mathrm{C}$ statistics respectively.

Results: Institutional overall mortality was $2.52 \%$. The expected mortality rates of SinoSCORE,

EuroSCORE II and STS risk evaluation system were 0.78(0.64)\%, 1.43(1.14)\% and 0.78(0.77)\%, younger group. SinoSCORE, EuroSCORE II and STS risk evaluation system all achieved positive calibrations in the entire cohort and subsets.

Conclusion: The performance of the three risk evaluation systems was not ideal in the entire cohort. In the risk evaluation system. 


\section{INTRODUCTION}

The number of cardiac surgeries in China is increasing steadily, especially coronary artery bypass

50 grafting $(\mathrm{CABG})$ [1]. Cardiac surgeries are currently regarded as safe and effective owing to the

51 development of surgical, anesthetic and perioperative management [2]. Preoperative risk evaluation

52 systems play an important role in current cardiac surgical practice. During the last two decades, various

53 risk evaluation systems have been developed to predict mortality for cardiac surgery, such as the European

54 System for Cardiac Operative Risk Evaluation (EuroSCORE) [3, 4] and the Society of Thoracic Surgeons

55 (STS) score [5]. In China, Sino System for Coronary Operative Risk Evaluation (SinoSCORE) [6, 7],

56 based on more than 9,000 Chinese patients was published in 2010.

57 Previous studies only evaluated the predictive capacity of these risk evaluation systems among

58 different operations and had rarely evaluated the ability to predict mortality among different age groups

59 [8-12]. Therefore, our study attempts to analyze the predictive capacity of the three risk evaluation

60 systems for patients aged $\geq 70$ who were treated with isolated CABG operation in East China. 


\section{METHODS}

\section{Patients}

Form January 2004 to September 2016, 2070 patients from two hospitals (the first affiliated underwent isolated CABG were invited to participate in the study, which was approved by ethic committees of the two hospitals (Ethical Application Ref: 2017-SR-053; [2017] research (018), respectively). All patients had been discharged when data was extracted. Inclusion criteria were isolated

CABG while exclusion criteria were CABG combined with other cardiac surgeries and missing of information. All enrolled patients signed informed consent forms. There were 124 (5.99\%) patients operative risk was predicted by the algorithms online SinoSCORE available at $h t t p: / / w w w . c v s-$

china.com/sino.asp, EuroSCORE II available at http://www.euroscore.org/calc.html and STS risk rates of each patient were ascertained by every system above. rate of CAD has increased significantly in people over 70 years old. To explore the predictive efficacy of the three evaluation systems, in each set, patients were divided into two subsets according to their age: elderly group (age $\geq 70,33.04 \%, 643 / 1946$ ) and younger group (age $<70,66.96 \%, 1303 / 1946$ ). The 
83 expected and observed mortality rates in entire cohort and each subset, respectively.

\section{Outcome Endpoint}

85 The outcome of interest in this study was in-hospital mortality, which was defined as postoperative

86 in-hospital all-cause death. 


\section{Statistical Analysis}

88 Statistical analysis was performed with SPSS version 19.0 (SPSS Inc., Chicago, Illinois, USA). If

89 continuous variables satisfy the normal distribution, then variables were expressed as mean \pm standard

90 deviation, else variables were expressed as median and interquartile range (IQR). Categorical variables

91 were expressed as percentages. Statistical analysis comparing groups was performed using t-tests for

92 continuous variables, Mann-Whitney-Wilcoxon tests for ordinal or continuous variables that do not satisfy

93 the assumptions required for a t-test, and Fisher's exact or $\chi 2$ (chi-square) tests for categorical variables. A

94 P-value of less than 0.05 was considered significant.

95 Performance of the three risk evaluation systems was assessed by comparing the expected and

96 observed in-hospital mortality rates. Calibration (statistical precision) was analyzed by Hosmer-

97 Lemeshow (H-L) goodness-of-fit statistic [13]. The H-L statistic measured the differences between

98 expected and observed outcomes. P-value greater than 0.05 means there is no evidence that this risk

99 evaluation system is poorly calibrated. The area under the receiver operating characteristic curve (AUC),

100 which was used to assess how well the evaluated system could discriminate between survivors and non-

101 survivors, describes an estimate of the model's discrimination ability [14]. The discriminative power of

102 the risk evaluation system is considered excellent if the AUC $>0.80$, good if $>0.75$ and acceptable if $>0.70$.

103 Calibration plots of observed versus expected mortality rates for 20 equally sized groups by ranked

104 expected rates calculated of the three systems were constructed. The ideal calibrated predictions consist of

105 a $45^{\circ}$ line, Where points below or above the diagonal line indicated overestimation or underestimation

106 respectively. Plots of observed and expected mortality rates of the three systems by age distribution were

107 also drawn, to illustrate the differences mortality rate in subsets.

108 Finally, the net benefit of three risk evaluation systems for predicting in-hospital mortality was 
109 performed by Decision Curve Analysis (DCA). DCA consisted in the subtraction of the proportion of all

110 patients who are false-positive from the proportion who are true-positive, weighting by the relative harm

111 of a false-positive and a false-negative result. DCA was performed using R software version 3.4.0 (The R

112 Foundation for Statistical Computing; State of Jersey, Austria) with package Decision curve. 
113 RESULTS

114 Performance in Elderly Group

115 There were 31 observed deaths, with an observed mortality rate of $4.82 \%$ (Table 1). Expected 116 mortality rate of SinoSCORE, EuroSCORE II and STS risk evaluation system for the elderly group were $1171.06(0.87) \%, 2.21(1.29) \%$ and $1.27(0.97) \%$, respectively. The expected ability of the three risk evaluation 118 systems in the elderly group is shown in Table 2. SinoSCORE, EuroSCORE II and STS risk evaluation system all showed positive calibration in predicting in-hospital mortality $(\mathrm{H}-\mathrm{L}: \mathrm{P}=0.053, \mathrm{P}=0.389$ and $\mathrm{P}=0.061$, respectively) (Table 2). The discriminative power for the elderly group in SinoSCORE was the best (0.829), followed by STS risk evaluation system (0.730) and EuroSCORE II (0.640) (Fig. 1). the selected threshold, included between 0 and 20\% (Fig. 2).

\section{Performance in younger group}


135 showed positive calibration (H-L: $\mathrm{P}=0.643, \mathrm{P}=0.527$ and $\mathrm{P}=0.321$, respectively) (Table 2). The

136 discriminative power for the younger group in EuroSCORE II was the highest (0.785), followed by STS

137 risk evaluation system (0.772) and SinoSCORE (0.769) (Table 2, Fig. 1). Three risk evaluation systems all

138 showed good discrimination in the younger group. The decision curve of SinoSCORE, EuroSCORE II and

139 STS risk evaluation system remained very close regardless of the threshold selected. The benefit of

140 EuroSCORE II was relatively greater than that of SinoSCORE and the STS risk evaluation system

141 between 0 and $20 \%$ (Fig. 2).

142 Patient Baseline Data

143 Of the 1946 patients, the median age was $66.00(11.00)$ years, $643(33.04 \%)$ were aged $\geq 70$ years old, $144396(20.35 \%)$ were female, and median left ventricular ejection fraction (LVEF) was $63.00(6.00) \%$. There 145 were 49 observed deaths, with an overall observed mortality rate of $2.52 \%$ (Table 3 ). The elderly group 146 was more likely to have higher serum creatinine $(\mathrm{P}<0.001)$, chronic obstructive pulmonary disease 147 (COPD) $(\mathrm{P}=0.001)$, The New York Heart Association (NYHA) class IV $(\mathrm{P}=0.013)$, preoperative intra148 aortic balloon pump (IABP) $(\mathrm{P}=0.040)$, and hospital mortality $(\mathrm{P}<0.001)$. The younger group had higher 149 weight $(\mathrm{P}<0.001)$, body mass index $(\mathrm{P}<0.001)$, body surface area $(\mathrm{P}<0.001)$, endogenous creatinine 150 clearance rate $(\mathrm{P}<0.001)$, proportionately more grafts $(\mathrm{P}<0.001)$ and Number of grafts $(\mathrm{P}<0.001)$. (Table 151 1)

152 Expected mortality mean mortality percentage of SinoSCORE, EuroSCORE II and STS risk 153 evaluation system for the entire cohort were $0.78(0.64) \%, 1.43(1.14) \%$ and $0.78(0.77) \%$, respectively. The 154 expected abilities of the three risk evaluation systems in entire cohort were shown in Table 2. 155 SinoSCORE, EuroSCORE II and STS risk evaluation system all showed positive calibration in predicting 156 in-hospital mortality (H-L: $\mathrm{P}=0.411, \mathrm{P}=0.113$ and $\mathrm{P}=0.230$, respectively) (Table 2 ). The discriminative 
157 power for the entire cohort (AUC) in SinoSCORE was the best (0.829), followed by STS risk evaluation 158 system (0.790) and EuroSCORE II (0.769) (Fig. 3).

159 Calibration plots showed that the three risk evaluation systems deviated from the diagonal line, so 160 these risk evaluation systems all underestimated mortality rates in the entire cohort. (Fig. 4) When the age 161 factor was considered, the mortality obviously increased with age. SinoSCORE, EuroSCORE II and STS 162 risk evaluation system overestimated in-hospital mortality rates in patients under 55 years, but 163 underestimated in-hospital mortality rates in patients over 55 years. (Fig. 5) 


\section{DISCUSSION}

Preoperative risk assessment of patients undergoing CABG is critical for treatment decisions-making, prognostic judgments, preoperative patient education and quality-assurance measure [15]. The EuroSCORE II and STS risk evaluation system, which were widely used around the world, have been well evaluated in different countries and regions [11, 16-18]. However, they may not accurately predict preoperative risk of Chinese patients and they require validation before clinical application $[19,20]$. In China, based on more than 9,000 patients in a national multi-centre database known as the Chinese Coronary Artery Bypass Grafting Registry Study [6, 21], Sino System for Coronary Operative Risk Evaluation (SinoSCORE) was published in 2010 [7]. After its publication, SinoSCORE was accepted and achieved good assessments in several medical centres in China [22-24].

Patients from the two regional central hospitals in East China could represent typical East China patients. Additionally, there was no difference in surgical techniques. Any risk evaluation system could show its best performance, only when the patient's characteristics and treatment process were similar to those which the system was originated. Therefore, risk evaluation system should be tested in the local population before used reliably. So it is very meaningful to assess and compare the predictive ability of the three risk evaluation systems in East China patients, especially in the elderly. However, previous studies rarely evaluated the ability to predict mortality among subsets grouped by age.

In this study, SinoSCORE was superior to EuroSCORE II and STS risk evaluation system in predicting operative mortality both in entire cohort and in the elderly group. There are some possible reasons to explain the results: (1) SinoSCORE was based on Chinese patients, the characteristics of patients were similar to the study database; (2) Removing 41 cases involved in SinoSCORE, we compared the differences between our local database and SinoSCORE database. There were some similar risk 
186 factors between two databases, such as age, hypertension, diabetes, renal failure, peripheral vascular

187 disease, active endocarditis, critical preoperative state, three-vessel coronary disease and in-hospital

188 mortality $[6,7,21]$, and which might be the reason contributed to SinoSCORE's a good expected power

189 (Table 4); (3) SinoSCORE was designed for isolated CABG operation, while EuroSCORE II and STS risk

190 evaluation system were not only suitable for isolated CABG but also for other cardiac surgeries, such as

191 valvular surgery and aortic surgery. Therefore, SinoSCORE was more suitable in the study and showed

192 better expected performance.

193 Although the three systems all had positive calibration and discrimination, it was a pity that they

194 substantially underestimated the mortality in the entire cohort and subsets. The discrepancy between

195 observed and expected mortality was particularly high in the elderly group $(4.82 \% \mathrm{vs} .1 .06 \%, 2.21 \%$,

$1961.27 \%$, respectively). One reason may be related to the predictive accuracy of the three systems; another is

197 that the preoperative parameters of the elderly were different from those of normal population.

198 Aging is a global problem, China, as a developing country, is also facing this problem. Although

over 70 years old accounted for 33.04\%. Previous literature has reported that the proportion of patients

over 70 years was up to $60 \%$ [28]. Patients are now older, they have more comorbidities and more 

than the STS risk evaluation system (18 vs 26), thereby making it easier to use in clinical practices. two systems. Comparing the variables of the three systems, only a few were the same, such as age, diabetes, status of surgery and type of surgery, which could lead to the difference in predictive capabilities among them. countries was $2.18 \sim 2.50 \%$ [29-33]. The mortality rate in this study was $2.52 \%$, slightly higher than previous reported. It also explained that the three risk evaluation systems under estimated mortality rates.

One possible reason was that although cardiac surgery and perioperative care in China had developed rapidly in the last decades, there were still some gaps compared with the developed countries. Another possible reason was that there were 124 patients excluded from the study. The quality control of the hospital required all in-hospital deaths should be archived. As a result, this study included all deaths (except 2 death developing SinoSCORE); to some extent, the mortality rate had increased.

$$
\text { SinoSCORE solved the problem that China did not have its own heart surgery risk evaluation system. }
$$
shows no compromise. system can also predict other outcomes, evaluating the predictive capacities of STS risk evaluation system 
230 retrospective and non-randomized observational study. The sample size was still small compared with 231 other studies that were sourced from a large number of cases. The three risk evaluation systems 232 underestimated the mortality in the entire corhort and subsets in spite of their positive discrimination and 233 calibration. These factors might result in bias. Therefore, the mortality statistics may be limited to some 234 degree.

235 CONCLUSION

236 The performance of the three risk evaluation systems was not ideal, although the three risk 237 evaluation systems showed positive discrimination and calibration in the entire cohort. SinoSCORE 238 achieved slightly better predictive efficiency than STS risk evaluation system in elderly patients 239 underwent CABG in East China. 


\section{ACKNOWLEDGMENTS}

241 We wish to thank the help in surgeries given by Prof. Yongfeng Shao, Prof. Xiaowei Wang, Dr. Lei Wei,

242 Dr. Xiangxiang Zheng, Dr. Haoliang Sun. Dr. Luyao Ma, and Dr. Wei Zhang. 
Wang Y, Shi X, Du R, Chen Y, Zhang Q. 2016. Off-pump versus on-pump coronary artery bypass grafting in patients with diabetes: a meta-analysis. Acta diabetologica 54:1-10

2. Hu XL, Chen Y, Zhou ZD, Ying J, Hu YH, Xu GH. 2016. Posterior pericardiotomy for the prevention of atrial fibrillation after coronary artery bypass grafting: A meta-analysis of randomized controlled trials. International journal of cardiology 215:252

5. Fortescue EB, Kahn K, Bates DW. 2001. Development and validation of a clinical prediction rule for major adverse

6. Li Y, Zheng Z, Hu S. 2009. The Chinese coronary artery bypass grafting registry study: analysis of the national multicentre database of 9248 patients. Heart 95:1140 xue guan bing za zhi [Chinese journal of cardiovascular diseases] 38:901-904

10. Aggarwal N, Selvendran S, Newsome S, Malley T, Auger D, Prasad S, Vassiliou V. 2016. 43 Euroscore II and STS Risk Model Scores in Aortic Stenosis: Can We Rely on Them. Heart 102:A31-A32.

11. Ad N, Holmes SD, Patel J, Pritchard G, Shuman DJ, Halpin L. 2016. Comparison of EuroSCORE II, Original EuroSCORE, and The Society of Thoracic Surgeons Risk Score in Cardiac Surgery Patients. Annals of Thoracic Surgery 102:573-579

12. Garcia-Valentin A, Mestres CA, Bernabeu E, Bahamonde JA, Martín I, Rueda C, Domenech A, Valencia J, Fletcher D, Machado F. 2016. Validation and quality measurements for EuroSCORE and EuroSCORE II in the Spanish cardiac surgical population: a prospective, multicentre study. European journal of cardio-thoracic surgery: official journal of the European Association for Cardio-thoracic Surgery 19:2

13. Lemeshow S, Hosmer DW Jr. 1982. A review of goodness of fit statistics for use in the development of logistic regression models. American journal of epidemiology 115:92-106

14. Hanley JA, Mcneil BJ. 1982. The meaning and use of the area under a receiver operating characteristic (ROC) curve. Radiology 143:29-36

15. Patratdelon S, Rouxel A, Gacouin A, Revest M, Flécher E, Fouquet O, Le TY, Lerolle N, Tattevin P, Tadié JM. 2016. EuroSCORE II underestimates mortality after cardiac surgery for infective endocarditis. European journal of cardiothoracic surgery : official journal of the European Association for Cardio-thoracic Surgery 49:944

16. Aydın MS, Göz M, Hazar A, Koçarslan A. 2015. Comparison of EuroSCORE and STS risk parameters in patients performed with coronary artery bypass surgery. Dicle Medical Journal 42:1

17. Holinski S, Jessen S, Neumann K, Konertz W. 2015. Predictive Power and Implication of EuroSCORE, EuroSCORE II 
285

286

287

288

289

290

291

292

293

294

295

296

297

298

299

300

301

302

303

304

305

306

307

308

309

310

311

312

313

314

315

316

317

318

319

320

321

322

323

324

325

and STS Score for Isolated Repeated Aortic Valve Replacement. Annals of Thoracic \& Cardiovascular Surgery Official Journal of the Association of Thoracic \& Cardiovascular Surgeons of Asia 21:242-246

18. George J, George A, Bhat P, Bhat S, Manjunath CN. 2015. Combining SYNTAX score with EuroSCORE II to predict inhospital outcome after CABG surgery. Indian heart journal 67:S108-S108

19. Bai Y, Wang L, Guo Z, Chen Q, Jiang N, Dai J, Liu J. 2016. Performance of EuroSCORE II and SinoSCORE in Chinese patients undergoing coronary artery bypass grafting. Interactive Cardiovascular \& Thoracic Surgery 23:ivw224

20. Wang TK, Li AY, Ramanathan T, Stewart RA, Gamble G, White HD. 2014. Comparison of four risk scores for contemporary isolated coronary artery bypass grafting. Heart Lung \& Circulation 23:469-474

21. Zheng Z, Li Y, S, Hu S. 2009. The Chinese coronary artery bypass grafting registry study: how well does the EuroSCORE predict operative risk for Chinese population. European Journal of Cardio-Thoracic Surgery 35:54-58

22. Yu J, Wan F, Xie JY, Wu S, Cui ZQ, Xu M, Zhang Z, Gao W. 2015. [Role of EuroSCORE and SinoSCORE in prediction of early postoperative quality of life in patients after coronary artery bypass surgery]. Beijing da xue xue bao. Yi xue ban = Journal of Peking University. Health sciences 47:769-773

23. Feng L, Dai CY, Zhe-Xin LU, Bo X, Zhen-Lei HU, Huang RT, Gen-Xing XU, Song X. 2014. Comparison of SinoSCORE and EuroSCORE in prediction of early mortality in off-pump coronary artery bypass surgery. Journal of Shanghai Jiaotong University 34:165-168

24. Liu PM, Sang XM, Yuan GY, Hua P, Yang YQ, Wang JF. 2013. GW24-e3724 Comparison of the SinoSCORE and the EuroSCORE for predicting in-hospital mortality in patients undergoing coronary artery bypass Grafting. Heart 99:E155E155

25. Wu L, He Y, Jiang B, Sun D, Wang J, Liu M, Yang S, Wang Y. 2015. Trends in Prevalence, Awareness, Treatment and Control of Hypertension during 2001-2010 in an Urban Elderly Population of China. PloS one 10:e0132814

26. Li Q, Yang Z, Zhang W, Gu H, Lu S, Shi Q, Yin X, Li X, Li R, Ning G. 2015. Association between metabolic syndrome and lung function in middle-aged and elderly Chinese individuals. Diabetes Research \& Clinical Practice 108:e46-e48

27. Zhen D, Liu L, Guan C, Zhao N, Tang X. 2015. High prevalence of vitamin D deficiency among middle-aged and elderly individuals in northwestern China: Its relationship to osteoporosis and lifestyle factors. Bone 71:1-6

28. Churpek MM, Yuen TC, Winslow C, Hall J, Edelson DP. 2015. Differences in vital signs between elderly and nonelderly patients prior to ward cardiac arrest. Critical care medicine 43:816-822

29. Moazzami K, Dolmatova E, Maher J, Gerula C, Sambol J, Klapholz M, Waller AH. 2017. In-Hospital Outcomes and Complications of Coronary Artery Bypass Grafting in the United States Between 2008 and 2012. Journal of Cardiothoracic \& Vascular Anesthesia 31:19

30. Curtis M, Yi D, Lee VV, Elayda MAA, Coselli JS, Collard CD, Wei P. 2017. Effect of Dose and Timing of Preoperative Statins on Mortality After Coronary Artery Bypass Surgery. Annals of Thoracic Surgery

31. Swaminathan RV, Feldman DN, Pashun RA, Patil RK, Shah T, Geleris JD, Wong SC, Girardi LN, Gaudino M, Minutello RM. 2016. Gender Differences in In-Hospital Outcomes After Coronary Artery Bypass Grafting. American Journal of Cardiology 118:362-368

32. Herlitz J, Wognsen GB, Karlson BW, Sjland H, Karlsson T, Caidahl K, Hartford M, Haglid M. 2015. Mortality, mode of death and risk indicators for death during 5 years after coronary artery bypass grafting among patients with and without a history of diabetes mellitus. Coronary artery disease 11:339-346

33. Kuwaki K, Inaba H, Yamamoto T, Dohi S, Matsumura T, Morita T, Amano A. 2015. Performance of the EuroSCORE II and the Society of Thoracic Surgeons Score in patients undergoing aortic valve replacement for aortic stenosis. The Journal of cardiovascular surgery 56:455-462 
Fig 1. The receiver operating characteristic curves of the three risk evaluation systems with subsets

(A)the receiver operating characteristic curves of the three risk evaluation systems with elderly group of the three risk evaluation systems with younger group (SinoSCORE 0.769, EuroSCORE II 0.785 and STS 0.772)

Fig 2. DCA showed the clinical usefulness of SinoSCORE, EuroSCORE II and STS risk evaluation system in predicting in-hospital mortality

The gray line represents the net benefit of providing surgery for all patients, assuming that all patients would survive. The black line represents the net benefit of surgery to none patients, assuming that none would survive after surgery. The red, blue and green lines represent the net benefit of applying surgery to patients according to EuroSCORE II, SinoSCORE, and STS risk evaluation system, respectively. The selected probability threshold is plotted on the abscissa.

(A)DCA for elderly group.(B)DCA for younger group.

Fig 3. The receiver operating characteristic curves of three risk evaluation systems in total patients For all of total patients, the receiver operating characteristic curves of SinoSCORE is 0.829, of

EuroSCORE is 0.769 , and of STS risk evaluation system is 0.790 ,respectively.

Fig 4. Calibration plots for the three risk evaluation systems

Calibration plots for SinoSCORE, EuroCORE and STS. 


\section{Fig 5. Plots of observed and expected mortality rates of the three systems by age distribution}

345 Three risk evaluation systems overestimated in-hospital mortality rates in patients under 55 years, but

346 underestimated in-hospital mortality rates in patients over 55 years. 


\section{Table $\mathbf{1}$ (on next page)}

Baseline clinical characteristics of subgroups.

BMI, body mass index; COPD, chronic obstructive pulmonary disease; Scr, Serum creatinine; $\mathrm{Ccr}$, endogenous creatinine clearance rate; LVEF, left ventricular ejection fraction 
Table 1. Baseline clinical characteristics of subgroups.

\begin{tabular}{|c|c|c|c|}
\hline Risk factors & Elderly group $(n=643)$ & Younger group $(n=1303)$ & $P$-value \\
\hline Age (y) & $74.00(5.00)$ & $62.00(8.00)$ & $<0.001$ \\
\hline Female $(n, \%)$ & $135(20.96)$ & $261(20.03)$ & 0.619 \\
\hline Weight (kg) & $67.00(13.00)$ & $70.00(14.00)$ & $<0.001$ \\
\hline Height (cm) & $168.00(11.00)$ & $168.00(10.00)$ & 0.021 \\
\hline BMI (kg/m2) & $24.22(3.79)$ & $25.00(3.90)$ & $<0.001$ \\
\hline Morbid obesity (n,\%) & $22(3.42)$ & $76(5.83)$ & 0.022 \\
\hline Body surface area (m2) & $1.72 \pm 0.15$ & $1.76 \pm 0.16$ & $<0.001$ \\
\hline Diabetes (n,\%) & $196(30.48)$ & $395(30.31)$ & 0.940 \\
\hline Hypertension (n,\%) & $454(70.61)$ & $868(66.62)$ & 0.076 \\
\hline Renal failure (n,\%) & $9(1.40)$ & $11(0.84)$ & 0.253 \\
\hline Serum creatinine $(\mu \mathrm{mol} / \mathrm{l})$ & $82.00(27.30)$ & $74.90(24.00)$ & $<0.001$ \\
\hline $\operatorname{Ccr}(\mathrm{ml} / \mathrm{min})$ & $64.16(23.18)$ & $86.57(32.07)$ & $<0.001$ \\
\hline Stroke $(\mathrm{n}, \%)$ & $18(2.72)$ & $22(1.66)$ & 0.104 \\
\hline COPD (n,\%) & $24(3.73)$ & $19(1.46)$ & 0.001 \\
\hline Peripheral vascular disease $(\mathbf{n}, \%)$ & $18(2.80)$ & $25(1.92)$ & 0.214 \\
\hline Previous cardiac surgery (n,\%) & $27(4.20)$ & $42(3.22)$ & 0.274 \\
\hline Atrial flutter and fibrillation (n,\%) & $17(2.64)$ & $23(1.77)$ & 0.199 \\
\hline pulmonary hypertension (n,\%) & $56(8.71)$ & $100(7.67)$ & 0.728 \\
\hline Myocardial infarction (n,\%) & $74(11.21)$ & $180(13.56)$ & 0.167 \\
\hline Unstable angina pectoris $(\mathrm{n}, \%)$ & $388(58.79)$ & $726(54.71)$ & 0.096 \\
\hline Number of diseased vessels (n) & $3.00(0.00)$ & $3.00(0.00)$ & 0.082 \\
\hline Three-vessel coronary disease (n,\%) & $598(90.60)$ & $1167(87.94)$ & 0.101 \\
\hline NYHA IV (n,\%) & $20(3.03)$ & $17(1.28)$ & 0.013 \\
\hline LVEF (\%) & $63.00(5.80)$ & $63.10(6.00)$ & 0.425 \\
\hline Preoperative IABP (n,\%) & $10(1.56)$ & $5(0.38)$ & 0.040 \\
\hline Status of surgery & & & 0.999 \\
\hline Elective $(n, \%)$ & $611(95.02)$ & $1238(95.01)$ & \\
\hline Urgent (n,\%) & $25(3.89)$ & $51(3.91)$ & \\
\hline Salvage $(\mathbf{n}, \%)$ & $7(1.09)$ & $14(1.07)$ & \\
\hline Number of grafts (n) & $3.00(1.00)$ & $4.00(1.00)$ & $<0.001$ \\
\hline Hospital mortality (n,\%) & $31(4.82)$ & $18(1.38)$ & $<0.001$ \\
\hline
\end{tabular}

Abbreviations: BMI, body mass index; COPD, chronic obstructive pulmonary disease; Scr, Serum creatinine; Ccr, endogenous creatinine clearance rate; LVEF, left ventricular ejection fraction 


\section{Table 2 (on next page)}

Observed and expected mortality rates of the three systems.

AUC, area under receiver operating characteristic curve; H-L statistics, Hosmer-Lemeshow statistics 
Table2. Observed and expected mortality rates of the three systems.

\begin{tabular}{|c|c|c|c|c|c|c|c|c|c|}
\hline & $\begin{array}{c}\text { elderly group } \\
\text { of } \\
\text { SinoSCORE }\end{array}$ & $\begin{array}{c}\text { younger group } \\
\text { of } \\
\text { SinoSCORE }\end{array}$ & $\begin{array}{c}\text { total patients } \\
\text { of } \\
\text { SinoSCORE }\end{array}$ & $\begin{array}{c}\text { elderly group } \\
\text { of } \\
\text { EuroSCORE II }\end{array}$ & $\begin{array}{c}\text { younger group } \\
\text { of } \\
\text { EuroSCORE II }\end{array}$ & $\begin{array}{c}\text { total patients } \\
\text { of EuroSCORE } \\
\text { II }\end{array}$ & $\begin{array}{c}\text { elderly group } \\
\text { of } \\
\text { STS }\end{array}$ & $\begin{array}{c}\text { younger group } \\
\text { of } \\
\text { STS }\end{array}$ & $\begin{array}{c}\text { total patients } \\
\text { of } \\
\text { STS }\end{array}$ \\
\hline $\begin{array}{c}\text { Number of } \\
\text { patients }\end{array}$ & 643 & 1303 & 1946 & 643 & 1303 & 1946 & 643 & 1303 & 1946 \\
\hline Deaths & 31 & 18 & 49 & 31 & 18 & 49 & 31 & 18 & 49 \\
\hline $\begin{array}{c}\text { Observed } \\
\text { mortality(\%) }\end{array}$ & 4.82 & 1.38 & 2.52 & 4.82 & 1.38 & 2.52 & 4.82 & 1.38 & 2.52 \\
\hline $\begin{array}{c}\text { Expected } \\
\text { mortality(\%) }\end{array}$ & $1.06(0.87)$ & $0.67(0.50)$ & $0.78(0.64)$ & $2.21(1.29)$ & $1.18(0.70)$ & $1.43(1.14)$ & $1.27(0.97)$ & $0.60(0.50)$ & $0.78(0.77)$ \\
\hline AUC & 0.829 & 0.769 & 0.829 & 0.640 & 0.785 & 0.769 & 0.730 & 0.772 & 0.790 \\
\hline H-L statistics & 0.053 & 0.643 & 0.411 & 0.389 & 0.527 & 0.113 & 0.061 & 0.321 & 0.230 \\
\hline
\end{tabular}

Abbreviations:AUC, area under receiver operating characteristic curve; H-L statistics, Hosmer-Lemeshow statistics 


\section{Table 3(on next page)}

cardiac surgery patient baseline clinical characteristics.

BMI, body mass index; COPD, chronic obstructive pulmonary disease; Scr, Serum creatinine; $\mathrm{Ccr}$, endogenous creatinine clearance rate; LVEF, left ventricular ejection fraction. 
Table 3. cardiac surgery patient baseline clinical characteristics.

\begin{tabular}{|c|c|}
\hline Risk factors & Total $(n=1946)$ \\
\hline Age (y) & $66.00(11.00)$ \\
\hline Female $(\mathbf{n}, \%)$ & $396(20.35)$ \\
\hline Weight (kg) & $70.00(13.00)$ \\
\hline Height (cm) & $168.00(10.00)$ \\
\hline BMI (kg/m2) & $24.80(3.81)$ \\
\hline Morbid obesity (n,\%) & $98(5.04)$ \\
\hline Body surface area (m2) & $1.75 \pm 0.16$ \\
\hline Diabetes $(n, \%)$ & $591(30.37)$ \\
\hline Hypertension (n,\%) & $1322(67.93)$ \\
\hline Renal failure (n,\%) & $20(1.03)$ \\
\hline Serum creatinine $(\mu \mathrm{mol} / \mathrm{l})$ & $76.70(25.90)$ \\
\hline $\operatorname{Ccr}(\mathrm{ml} / \mathrm{min})$ & $78.47(33.34)$ \\
\hline Stroke (n,\%) & $40(2.06)$ \\
\hline $\operatorname{COPD}(\mathbf{n}, \%)$ & $43(2.21)$ \\
\hline Peripheral vascular disease (n,\%) & $43(2.21)$ \\
\hline Previous cardiac surgery (n,\%) & $69(3.55)$ \\
\hline Atrial flutter and fibrillation (n,\%) & $40(2.06)$ \\
\hline Pulmonary hypertension (n,\%) & $156(8.02)$ \\
\hline Myocardial infarction (n,\%) & $250(12.85)$ \\
\hline Unstable angina pectoris (n,\%) & $1086(55.81)$ \\
\hline Number of diseased vessels (n) & $3.00(0.00)$ \\
\hline Three-vessel coronary disease (n,\%) & $1729(88.85)$ \\
\hline NYHA IV (n,\%) & $34(1.75)$ \\
\hline LVEF (\%) & $63.00(6.00)$ \\
\hline Preoperative IABP (n,\%) & $43(2.21)$ \\
\hline \multicolumn{2}{|l|}{ Status of surgery } \\
\hline Elective $(\mathbf{n}, \%)$ & $1849(95.02)$ \\
\hline Urgent $(\mathbf{n}, \%)$ & $76(3.91)$ \\
\hline Salvage (n,\%) & $21(1.08)$ \\
\hline Number of grafts (n) & $3.00(1.00)$ \\
\hline Hospital mortality (n,\%) & $49(2.52)$ \\
\hline
\end{tabular}

Abbreviations: BMI, body mass index; COPD, chronic obstructive pulmonary disease; Scr, Serum creatinine; Ccr, endogenous creatinine clearance rate; LVEF, left ventricular ejection fraction. 


\section{Table 4(on next page)}

The baseline of SinoSCORE database and Local database. 
Table 4. The baseline of SinoSCORE database and Local database.

\begin{tabular}{|l|c|c|}
\hline Risk factors & SinoSCORE ( N=9248 ) & Local ( N=1946) \\
\hline Age (y) & $62.60 \pm 9.20$ & $65.28 \pm 8.33$ \\
\hline Female (\%) & 21.50 & 20.35 \\
\hline Diabetes (\%) & 26.40 & 30.37 \\
\hline Hypertension (\%) & 63.50 & 67.93 \\
\hline Renal failure (\%) & 0.60 & 1.03 \\
\hline Cerebrovascular accident (\%) & 8.30 & 2.06 \\
\hline COPD (\%) & 1.30 & 2.21 \\
\hline Peripheral vascular disease (\%) & 2.50 & 2.21 \\
\hline Previous cardiac surgery (\%) & 2.30 & 3.55 \\
\hline Active endocarditis(\%) & 0 & 0 \\
\hline Critical preoperative state(\%) & 4.60 & 5.50 \\
\hline Myocardial infarction (\%) & 9.60 & 12.85 \\
\hline Unstable angina pectoris (\%) & 31.10 & 55.81 \\
\hline Three-vessel coronary disease (\%) & 76.70 & 88.85 \\
\hline Emergency (\%) & 7.10 & 4.98 \\
\hline Pulmonary hypertension (\%) & 1.10 & 8.02 \\
\hline LVEF 30-50\% (\%) & 20.90 & 8.70 \\
\hline LVEF <30\% (\%) & 0.90 & 0.20 \\
\hline Isolated CABG(\%) & 87.80 & 100 \\
\hline Hospital mortality (\%) & 3.27 & 2.52 \\
\hline (\%) & & \\
\hline
\end{tabular}

Abbreviations: COPD, chronic obstructive pulmonary disease; LVEF, left ventricular ejection fraction. 


\section{Figure 1}

The receiver operating characteristic curves of the three risk evaluation systems with subsets

(A) The receiver operating characteristic curves of the three risk evaluation systems with elderly group (SinoSCORE 0.829, EuroSCORE II 0.640 and STS 0.730). (B) The receiver operating characteristic curves of the three risk evaluation systems with younger group (SinOSCORE 0.769, EuroSCORE II 0.785 and STS 0.772).
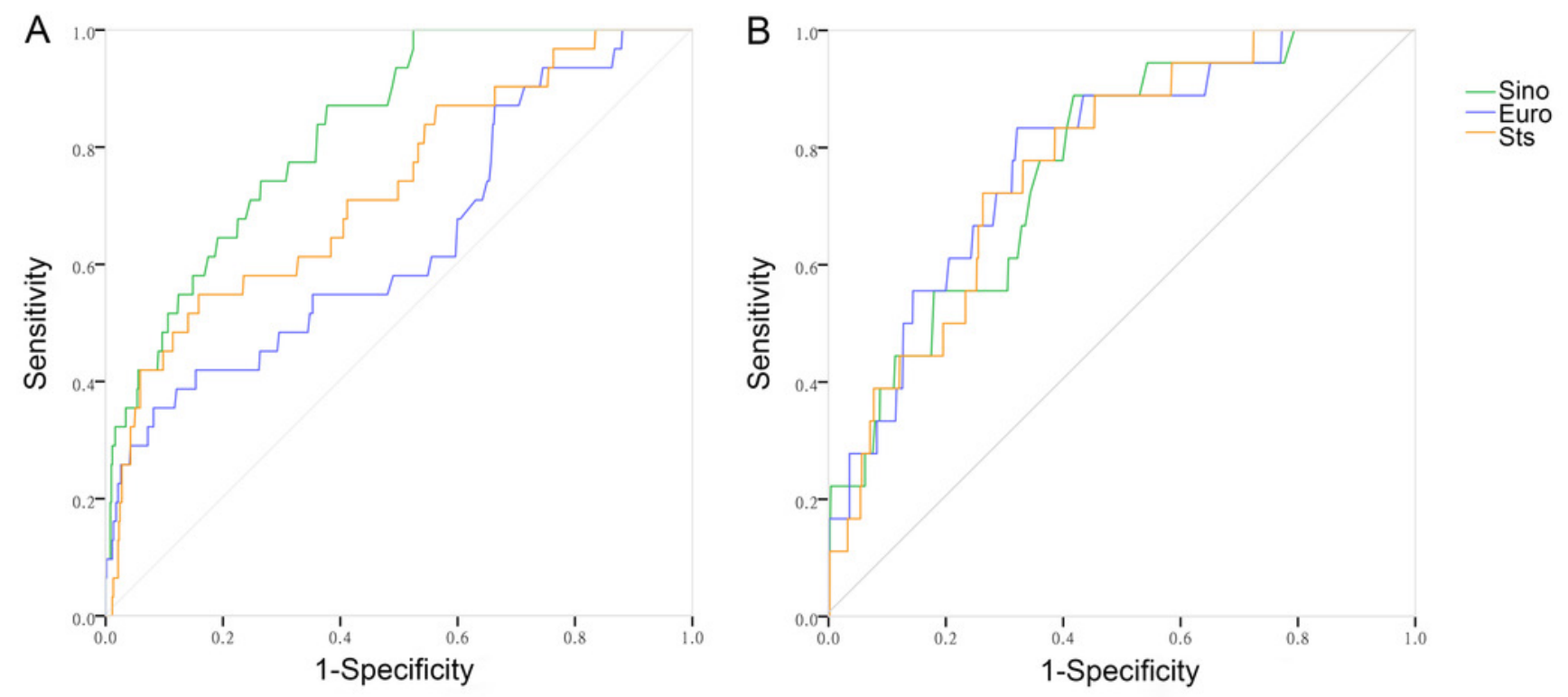


\section{Figure 2}

DCA showed the clinical usefulness of SinoSCORE, EuroSCORE II and STS risk evaluation system in predicting in-hospital mortality

The gray line represents the net benefit of providing surgery for all patients, assuming that all patients would survive. The black line represents the net benefit of surgery to none patients, assuming that none would survive after surgery. The red, blue and green lines represent the net benefit of applying surgery to patients according to EuroSCORE II, SinoSCORE, and STS risk evaluation system, respectively. The selected probability threshold is plotted on the abscissa.(A)DCA for elderly group.(B)DCA for younger group. 

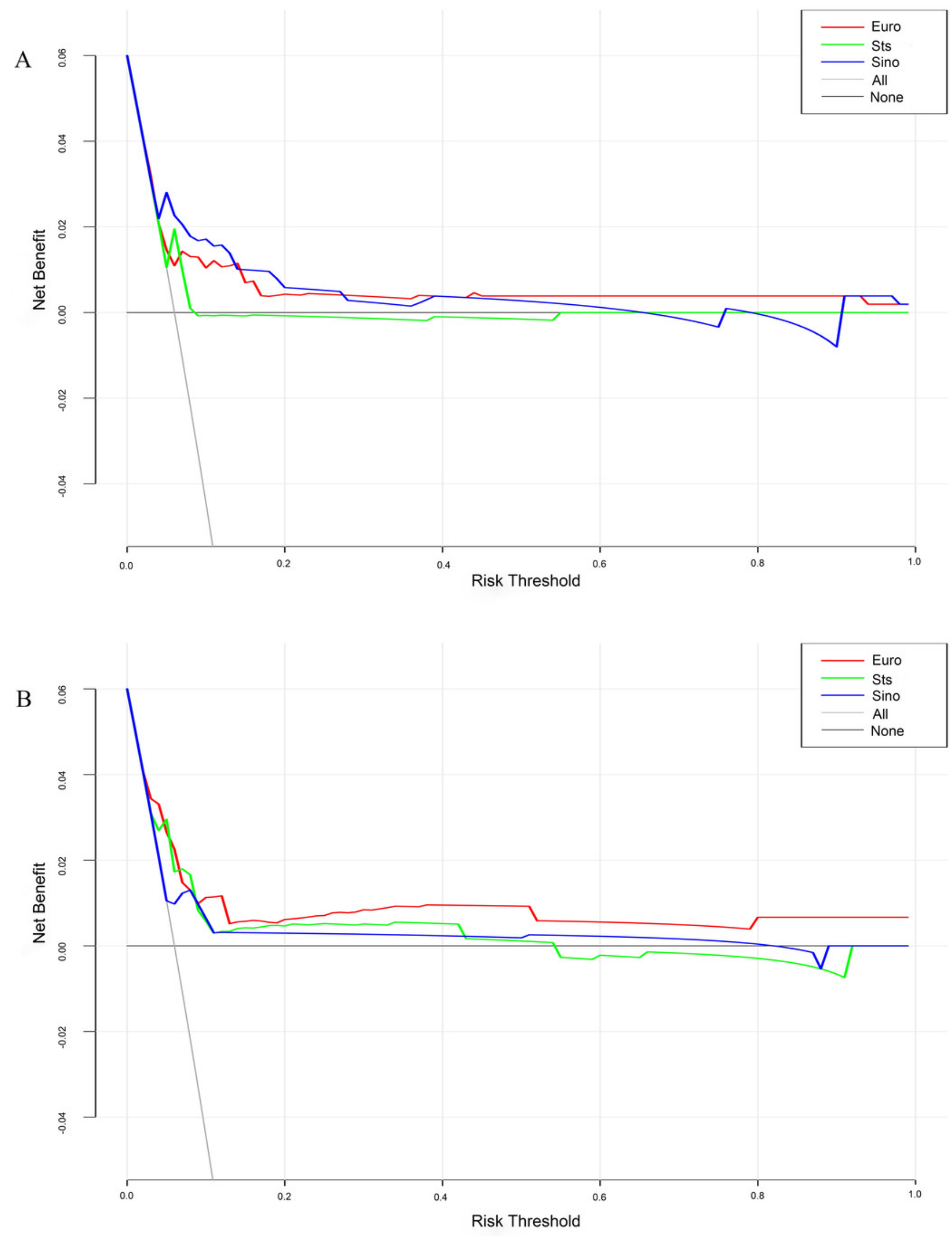
Figure 3

The receiver operating characteristic curves of three risk evaluation systems in total patients

For all of total patients, the receiver operating characteristic curves of SinoSCORE is $0.829[$ of EuroSCORE is 0.769 , and of STS risk evaluation system is 0.790 , respectively.

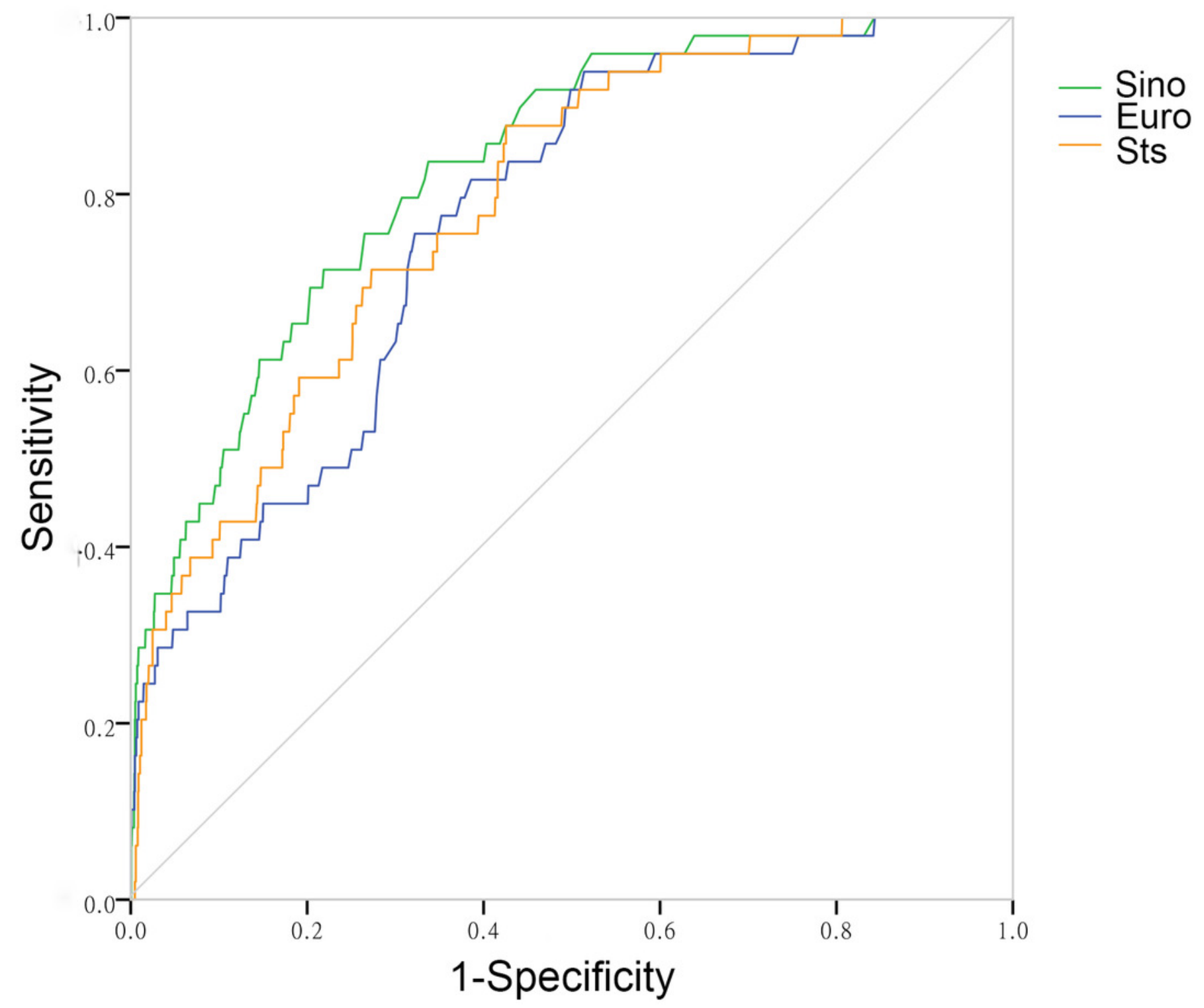


Figure 4

Calibration plots for the three risk evaluation systems

Calibration plots for SinoSCORE, EuroCORE and STS.

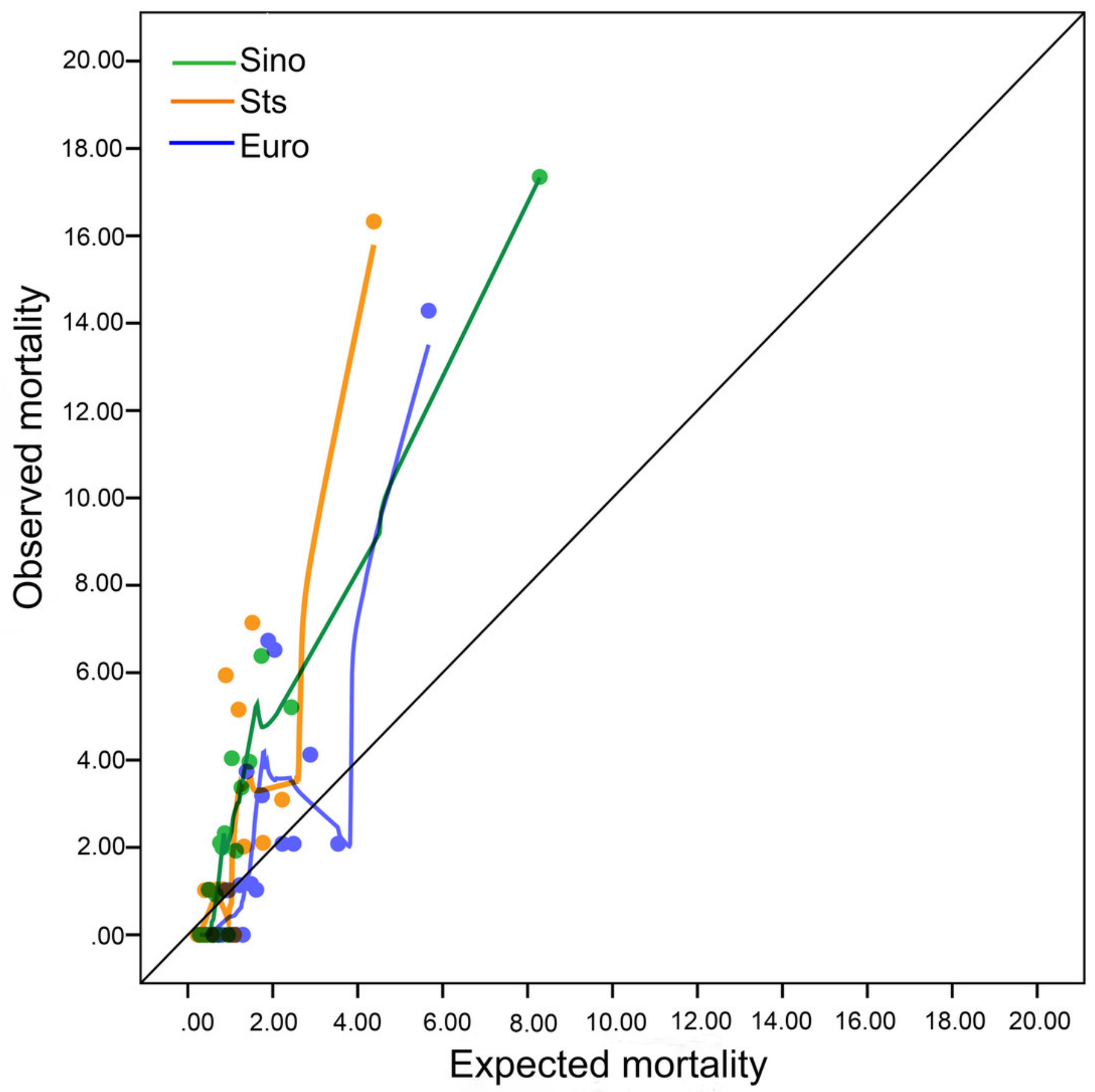


Figure 5

Plots of observed and expected mortality rates of the three systems by age distribution Three risk evaluation systems overestimated in-hospital mortality rates in patients under 55 years, but underestimated in-hospital mortality rates in patients over 55 years.

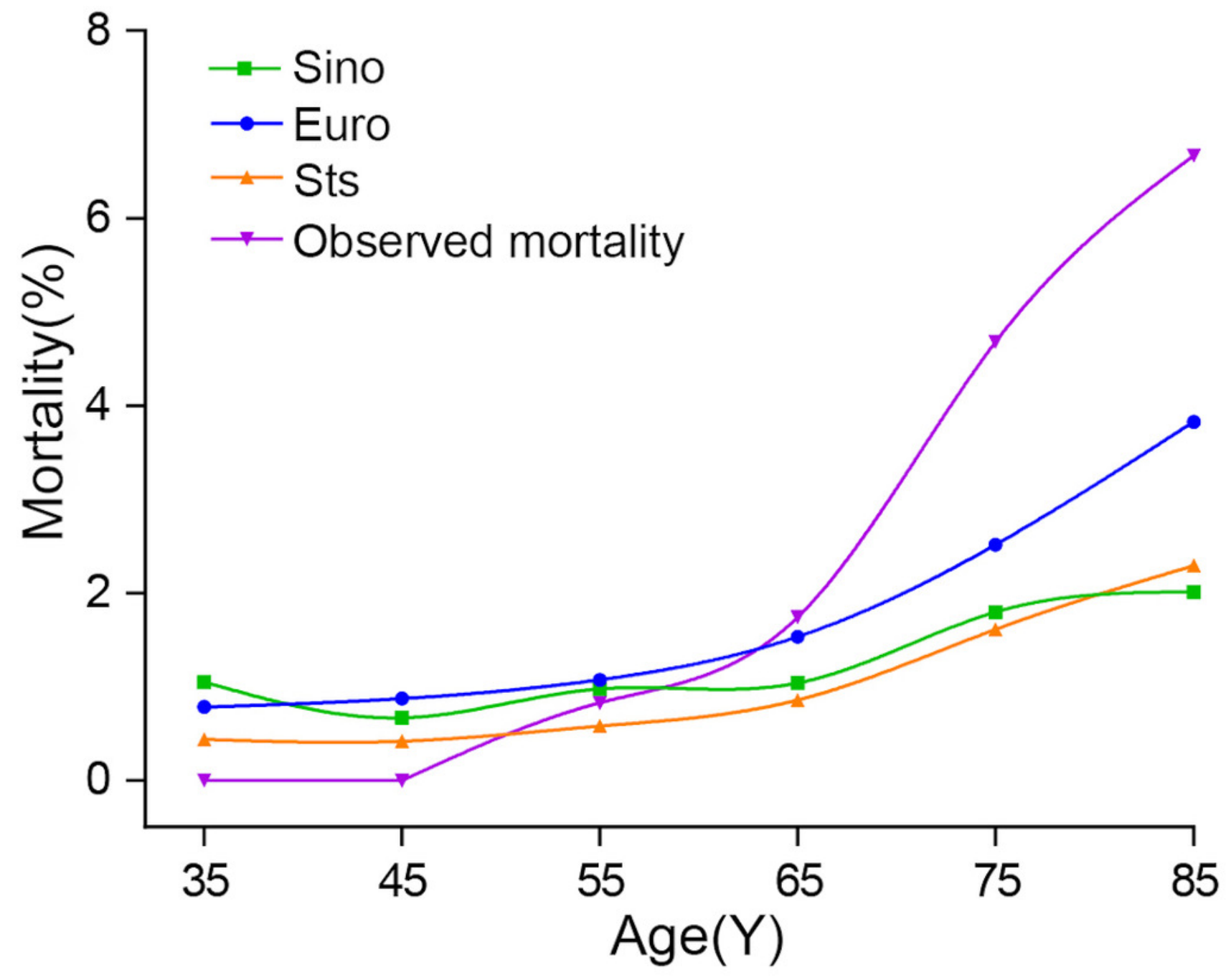

